\title{
Molecular genetic diversity and maternal origin of Chinese black-bone chicken breeds
}

\author{
W.Q. Zhu', ${ }^{1,2}$ H.F. Li' ${ }^{2}$, J.Y. Wang', J.T. Shu', C.H. Zhu' ${ }^{2}$, W.T. Song', \\ C. Song' ${ }^{2}$, G.G. Ji' ${ }^{2}$ and H.X. Liu ${ }^{2}$ \\ ${ }^{1}$ Jiangsu Institute of Poultry Science, Yangzhou, Jiangsu, China \\ ${ }^{2}$ Animal Science and Technology College, Yangzhou University, Jiangsu, China \\ Corresponding author: H.F. Li \\ E-mail: 1hfxf_002@ailiyun.com
}

Genet. Mol. Res. 13 (2): 3275-3282 (2014)

Received February 20, 2013

Accepted July 4, 2013

Published April 29, 2014

DOI http://dx.doi.org/10.4238/2014.April.29.5

\begin{abstract}
Chinese black-bone chickens are valued for the medicinal properties of their meat in traditional Chinese medicine. We investigated the genetic diversity and systematic evolution of Chinese black-bone chicken breeds. We sequenced the DNA of $520 \mathrm{bp}$ of the mitochondrial cyt $b$ gene of nine Chinese black-bone chicken breeds, including Silky chicken, Jinhu black-bone chicken, Jiangshan black-bone chicken, Yugan black-bone chicken, Wumeng black-bone chicken, Muchuan black-bone chicken, Xingwen black-bone chicken, Dehua black-bone chicken, and Yanjin black-bone chicken. We found 13 haplotypes. Haplotype and nucleotide diversity of the nine black-bone chicken breeds ranged from 0 to 0.78571 and 0.00081 to 0.00399 , respectively. Genetic diversity was the richest in Jinhu black-bone chickens and the lowest in Yanjin black-bone chickens. Analysis of phylogenetic trees for all birds constructed based on hyplotypes indicated that the maternal origin of black-bone chickens is predominantly from three subspecies of red jungle fowl. These results provide basic data useful for protection of black-bone chickens and help determine the origin of domestic chickens.
\end{abstract}

Key words: Chinese black-bone chicken; cyt b; Genetic diversity; Maternal origin 


\section{INTRODUCTION}

Mitochondrial cytochrome b (cyt b) is one of the proteins composing the mitochondrial complex III of the oxidative phosphorylation system and the only protein coded by mtDNA. With the characteristics of maternal inheritance and moderate rate of evolution in mtDNA, a short segment of the cyt $b$ sequence includes phylogenetic information from species to genera, even to class. The cytochrome $b$ gene has become the ideal marker molecule for studying the evolution and genetic diversity of animals.

The black-bone chicken breed, belonging to Galliformes, juvenile Branch, has special economic value in Chinese poultry breeds. To fully develop and utilize this valuable resource, many studies have focused on the genetic characteristics of black-bone chicken and improvement of their production performance (Zhou et al., 2006). A variety of different black-bone breeds have been developed, because of a vast territory, diverse environment, different selection targets and rearing conditions, and geographic proximity. It is, therefore, necessary to investigate these genetic resources with the aim of protection and development (Li and Qiu, 2003).

RAPD (Zhang et al., 1998a,b) and microsatellite marker methods (Sun et al., 2003; Zhu and Li, 2003; Tang et al., 2005; Li and Zhu, 2006) have been applied in most research related to the genetic diversity and population structure of black-bone chicken in the past. With the development of DNA sequencing techniques and extensive use of mtDNA molecular marker in studies on animal population genetic structure and systematic evolution, more and more studies have recently been reported on the genetic diversity, genetic differentiation and origin of chickens (Liu et al., 2006; Song et al., 2007; Zhu et al., 2009), ducks (He et al., 2008; Li et al., 2010) and geese (Wang et al., 2005; Li and Wang, 2007; Zhu et al., 2010), as well as other poultry by using mtDNA markers. However, there has been no systematic and precise report on genetic diversity and maternal origin of Chinese black-bone chicken using mtDNA molecular markers. Few studies on the genetic diversity and systematic evolution of Chinese black-bone chickens have been reported by Fu and Ruan (2002), Yang et al. (2004) and Huang et al. (2010). In this study, we collected 73 chickens from nine Chinese native black-bone chicken breeds, sequenced a 520-bp portion of the cyt b gene of these chickens, combined with the sequences those of Gallus gallus gallus, Gallus gallus bankiva, Gallus gallus spadiceus, Gallus lafayettei, Gallus sonneratti, and Gallus varius reported in GenBank, and then constructed a phylogenetic tree for these birds. This research provides basic data for protecting the genetic resources of black-bone chickens, and this information may be helpful in solving the maternal origins of these special chickens.

\section{MATERIAL AND METHODS}

Blood was sampled from chicken wing veins and collected in test tube containing anticoagulant solution. Abbreviated breed names of Yanjin black-bone chicken, Silky black-bone chicken, Yugan black-bone chicken, Jinhu black-bone chicken, Jiangshan black-bone chicken, Muchuan black-bone chicken, Wumeng black-bone chicken, Xingwen black-bone chicken and Dehua black-bone chicken were YJ, SM, YG, JH, JS, MC, WM, XW, and DH respectively. More details about the nine black-bone chicken breeds are shown in Table 1. The sex ratio of each breed was ranged from 3 males to 4 females to 1 male to 1 male. The genetic relationship between chickens should be avoided as much as possible. Twelve homologous mtDNA 
cyt b sequences of red jungle fowl were also collected including: G. g. gallus [AP003322, AB0044986] in Indochina, Thailand, and Sumtara; G. g. bankiva [AP003323, AB0044985] in Indonesia and Java; G. g. spadiceus [AB044987, AP003321] in west and southwest Yunnan of China, Indochina, Burma, and northern Malaysia; G. lafayettei [AB044990, AP003325]; G. sonneratti [AB044989, AP006741]; G. varius [AB044988, AP003324].

\begin{tabular}{lcll}
\multicolumn{5}{l}{ Table 1. Conservation status of 9 black-bone chicken breeds. } \\
\hline Breed & Size & Conservation type & Location \\
\hline YJ & 7 & Conservation zone & Yanjin country in Yunnan Province \\
SM & 8 & Conservation zone & Taihe country in Jiangxi Province \\
YG & 8 & Conservation zone & Yugan country in Jiangxi Province \\
JH & 8 & Conservation zone & Taining country in Fujian Province \\
JS & 9 & Conservation zone & Jiangshan city in Zhejiang Province \\
MC & 8 & Conservation zone & Muchuan country in Sichuan Province \\
WM & 7 & Conservation zone & Bijie in Guizhou Province \\
XW & 9 & Conservation zone & Xingwen country in Sichuan Province \\
DH & 7 & Conservation zone & Dehua country in Fujian Province \\
\hline
\end{tabular}

YJ, SM, YG, JH, JS, MC, WM, XW, DH represented Yanjin black-bone chicken, Silky black-bone chicken, Yugan black-bone chicken, Jinhu black-bone chicken, Jiangshan black-bone chicken, Muchuan black-bone chicken, Wumeng black-bone chicken, Xingwen black-bone chicken and Dehua black-bone chicken respectively.

DNA was isolated from blood and extracted by the phenol/chloroform method (Sambrook et al., 1989). Polymerase chain reaction (PCR) was performed to amplify part of the mtDNA cyt $b$. The primers reported by Xiang et al. (2000) were used to amplify the target region. The corresponding sequences were (L15218) CCTATACTATGGCTCCTACCT and (H15754) GCTAGTACGCCTCCGAGTTT. PCR was carried out on an Eppendorf Mastercycle. The reaction mixture contained $2.5 \mu \mathrm{L} 10 \mathrm{X}$ Buffer, $2.5 \mu \mathrm{L}$ dNTPs $(2.5 \mathrm{mM}), 2.5 \mu \mathrm{L}$ $\mathrm{Mg}^{2+}(25 \mathrm{mM}), 1 \mu \mathrm{L}$ each primer $(25 \mathrm{pmol} / \mu \mathrm{L}), 3.0 \mu \mathrm{L}$ genomic DNA $(50 \mathrm{ng} / \mu \mathrm{L})$, and 0.2 $\mu \mathrm{LTaq}$ polymerase $(5 \mathrm{U} / \mu \mathrm{L})$. The thermal cycling profile for mtDNA was preheating for $5 \mathrm{~min}$ at $95^{\circ} \mathrm{C}$, followed by 35 cycles of $45 \mathrm{~s}$ at $94^{\circ} \mathrm{C}, 45 \mathrm{~s}$ at 51 to $53^{\circ} \mathrm{C}$, and $1 \mathrm{~min}$ at $72^{\circ} \mathrm{C}$, a final extension of $10 \mathrm{~min}$ at $72^{\circ} \mathrm{C}$, and storage at $4^{\circ} \mathrm{C}$. PCR products were purified on an agarose gel and sequenced on an ABI Prism 3730 DNA Analyzer in both directions by primer walking using the BigDye Terminator V. 3.1 Cycle Sequencing kit (ABI, Foster City, CA, USA).

Electropherograms were obtained using the program "Chromas 1.45" and manually checked, insuring the veracity of the DNA sequences. Sequence alignments were performed using DNAman (version 6.0.40). Haplotype numbers, nucleotide variable sites, haplotype diversity and nucleotide diversity (Nei, 1982) were calculated using DnaSP version 4.10.7 (Rozas et al., 2003). Ignoring insertion/deletion mutations, the same sequences were considered to be one haplotype. Kimura 2-parameter distances between breeds were estimated in Mega version 3.1 (Kumar et al., 2004) and a neighbor-joining tree was then constructed. A medianjoining network of the mtDNA cyt $b$ sequence haplotypes was constructed according to Bandelt et al. (1999) using the program Network 4.5.0.1.

\section{RESULTS AND DISCUSSION}

The average nucleotide composition was $24.6 \%$ thymine $(\mathrm{T}), 36.5 \%$ cytosine $(\mathrm{C})$, $26.2 \%$ adenosine $(A)$ and $12.7 \%$ guanine $(\mathrm{G})$ in the 520 -bp fragment of mtDNA cyt b of 71 
black-bone chickens. The average percentage of $\mathrm{A}+\mathrm{T}$ content $(50.8 \%)$ was higher than $\mathrm{G}+\mathrm{C}$ $(49.2 \%)$. There were 17 polymorphic sites, 8 singleton polymorphic sites at $96,143,249,291$, $428,429,457$, and 467 sites and 9 parsimony informative polymorphic sites at 77, 137, 146, $155,191,276,399$, and 452 sites, respectively. The variable types were transitions and transversions, and no insertions or deletions were found.

Thirteen haplotypes were identified in nine black-bone chicken breeds. Ratio of haplotypes, haplotype diversity and nucleotide diversity of the nine black-bone chicken breeds ranged from 14 to $50 \%, 0$ to 0.78571 and 0 to 0.00399 , respectively (Table 2). This indicated that the genetic diversity between nine Chinese black-bone chicken breeds was significantly different and was not rich. Jinhu black-bone chicken had the highest values of ratio of haplotypes (50\%), haplotype diversity (0.78571) and nucleotide diversity (0.00399), and Yanjin black-bone chicken the lowest values of ratio of haplotypes (14\%), haplotype diversity (0) and nucleotide diversity (0), where these breeds had the richest and lowest genetic diversity, respectively. The phylogenetic tree results (Figures 1 and 2) showed that most haplotypes were close to red jungle fowl (H14-H19). The haplotypes H4 (YG3, JH3), $\mathrm{H} 5(\mathrm{JH} 1)$ and $\mathrm{H} 8 \mathrm{(MC1}$ ) were first clustered with $\mathrm{H} 21$ into a clade, and then clustered with other domestic chickens and red jungle fowl. Our results suggested that the maternal origin of black-bone chicken may overwhelmingly originate from three subspecies of red jungle and a few black-bone chickens may be a mix of a few greylag lineage chickens.

\begin{tabular}{lccccc}
\multicolumn{6}{l}{ Table 2. Haplotype diversity (Hd) and nucleotide diversity (Pi) in nine black-bone chicken breeds. } \\
\hline Breed & Sample size & No. of Haplotypes & Ratio of haplotypes (\%) & Haplotype diversity (Hd) & Nucleotide diversity (Pi) \\
\hline DH & 7 & 2 & 0.28571 & 0.28571 & 0.00275 \\
SY & 8 & 3 & 0.37500 & 0.46429 & 0.00145 \\
YG & 8 & 2 & 0.25000 & 0.53571 & 0.00103 \\
JH & 8 & 4 & 0.50000 & 0.78571 & 0.00399 \\
JS & 9 & 4 & 0.44444 & 0.58333 & 0.00332 \\
MC & 8 & 3 & 0.37500 & 0.46429 & 0.00145 \\
WM & 7 & 3 & 0.42857 & 0.52381 & 0.00165 \\
XW & 9 & 3 & 0.33333 & 0.41667 & 0.00128 \\
YJ & 7 & 1 & 0.14286 & 0.00000 & 0.00000 \\
\hline
\end{tabular}

Abbreviations of black-bone chicken breed name were the same as Table 1 stated.

\section{Genetic diversity of Chinese black-bone chicken breeds}

Generally speaking, genetic diversity means the sum of genetic information carried by all living creatures on earth. However, genetic diversity means genetic variation of different individuals between different colonies in intraspecies or within the same group. The richer the genetic diversity of a breed is, the stronger its adaptive capability to a changing environment will be. Haplotype diversity $(\mathrm{Hd})$ and nucleotide diversity (Pi) of populations are the main indices for evaluating mtDNA variation and genetic diversity of a breed or a population. The greater the values of $\mathrm{Hd}$ and $\mathrm{Pi}$ are, the richer the genetic diversity will be. Pi of nine blackbone chicken breeds $(0.188 \%)$ was significantly less than for Chinese native chicken $(1.8 \%)$ (Bao et al., 2008), and compared to other animals, Pi was higher than for domestic duck $(0.115 \%)$ (Li et al., 2010) and swine (0.122\%) (Lan et al., 1995) and significantly lower than for yak (1.231\%) (Lai et al., 2005) and scalper (2.16\%) (Liu et al., 2006). 


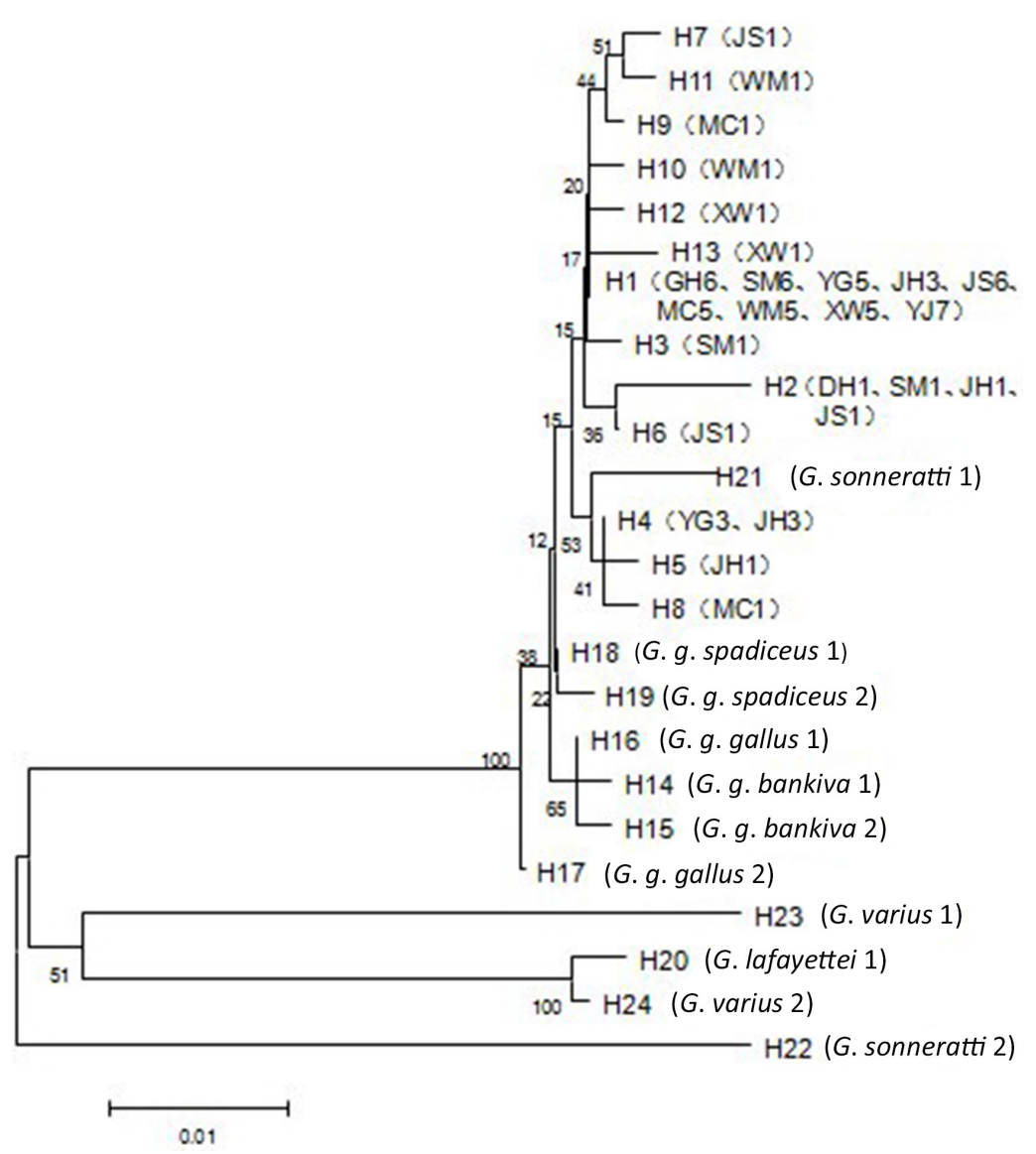

Figure 1. Phylogenetic tree of the cyt b sequences constructed with NJ method using Kimura's two parameter model. Key: $\mathrm{H}=$ haplotype. Abbreviations of black-bone chicken breed name were the same as Table 1 . The numbers after chicken breed name individuals sharing the same haplotype.

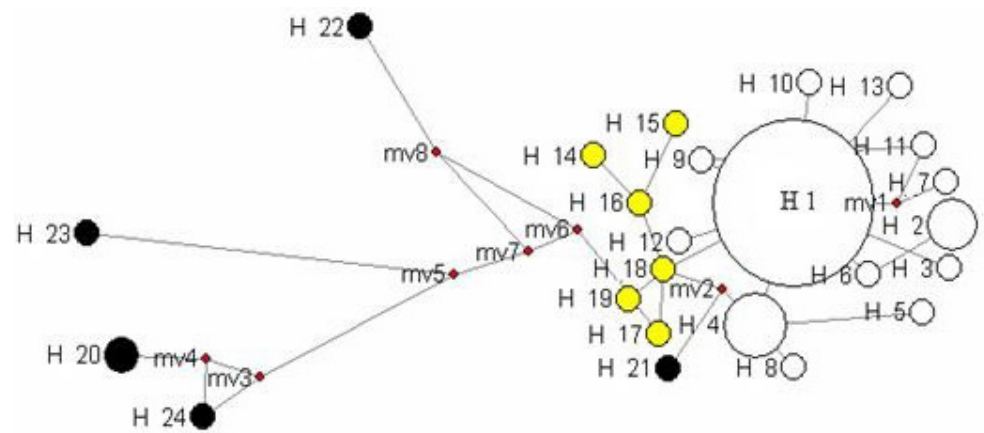

Figure 2. Reduced median-joining networks of mtDNA cyt b haplotypes. For haplotype codes see Figure 1. The red disk (mv1) represented the median vector which formed automatically in the Network software procedure. The detailed information of open, filled and yellow circles was the same with corresponding haplotype in Figure 1. 


\section{Maternal origins of Chinese black-bone chicken breeds}

The problem of the origin of domestic chickens has been the lack of a final conclusion at home and abroad. Darwin (1868) suggests that the red jungle fowl is the only ancestor of all domestic chicken, and pluralism scholars suggest that the red jungle fowl is the main ancestor and G. lafayettei, G. sonneratti, and G. varius are the secondary. In the last few years, although several authors have analyzed the origin of domestic fowl via mtDNA markers, there are still some differences according to the results. Fumihito et al. $(1994,1996)$ suggested that red jungle fowl, which was divided into island and mainland types because of its domestication in Thailand and surrounding areas, was ancestral and that the mainland type was the common ancestor of all domestic chickens.

However, recently Chinese researchers indicate that native domestic chickens originate from several subspecies of red jungle fowl. Niu et al. (2002) sequenced 539 bp of the mtDNA D-loop region of six Chinese native chicken breeds ( $G$. g. domesticus), and suggested that the Chinese domestic fowl probably originated from the red jungle fowl of Thailand and its adjacent regions, and indicated the egg breeds and general purpose chicken breeds may have originated from different maternal origins. Liu et al. (2006) reported that the domestic chicken across Eurasia may originate from different regions, such as Yunnan, South and Southwest China and/or surrounding areas (i.e., Vietnam, Burma, and Thailand), and the Indian subcontinent, which supports the theory 4 of multiple origins in South and Southeast Asia. Song et al. (2007) reported that six Chinese indigenous chicken breeds were clustered in three clades, of which clade A was dominant. Clade A included 17 haplotypes which were shared by ten Shouguang, nine Luxi game, five Wenchang, six Laiwu Black, six JiningBairi and two Langya individuals, accounting for $100,90,84,75,60$, and $20 \%$ of the total number of each breed, respectively. These indigenous chickens showed a close relationship with G. $g$. gallus, G. g. jabouile and G. g. spadiceus distributed in Laos and Yunnan Province in China. It indicated that the six Chinese indigenous chicken breeds may originate from the continental subspecies of G. gallus of Yunnan, Laos, and Vietnam or its surrounding areas. Gong et al. (2011) reported that Piao chickens had five maternal bloodlines. Zhu et al. (2009) suggested that Chinese game chickens originated from several subspecies of red jungle fowl and withstood a number of independent domestications. Jonas et al. (2008) reported that according to the skin color of domestic chicken and red jungle fowl and the evolution analysis of BCDO2 gene related to yellow skin, the yellow skin trait of most domestic chickens likely came from G. sonneratti. In other words, the domestic chickens were a mix of a few of the G. sonneratti lineage. In our study, the analysis of systematic evolution suggested that the maternal origin of black-bone chicken may overwhelmingly originate from three subspecies of red jungle fowl and that a few black-bone chickens may be a mix of greylag lineage chickens.

\section{CONCLUSION}

In conclusion, the genetic diversity of Chinese black-bone chicken is not rich and the special chickens may overwhelmingly originate from three subspecies of red jungle fowl, and a few black-bone chickens may be a mix of a few greylag lineage chickens. The results will provide reliable basic information for protecting genetic resources of Chinese black-bone chicken and for studying the origin of domestic chickens. 


\section{ACKNOWLEDGMENTS}

Research supported by the Earmarked Fund for Modern Agro-Industry Technology Research System, the National High Technology Research and the Development Program “863” (\#2011AA100301, \#2011AA100305), and the Introduction Project for Advanced International Scientific Technology of Agriculture (\#2011-Z65).

\section{REFERENCES}

Bandelt HJ, Forster P and Röhll A (1999). Median-joining networks for inferring intraspecific phylogenies. Mol. Biol. Evol. 16: 37-48.

Bao WB, Shu JT, Wang CB, Zhang HX, et al. (2008). Investigation on genetic diversity and systematic evolution in Chinese domestic fowls and red jungle fowls by analyzing the mtDNA control region. Acta Vet. Zootechnica Sin. 39: 1449-1459.

Darwin CR (1868). The Variation of Animals and Plants Under Domestication. John Murray, London. First edn., First issue. Volume 2.

Fu Y and Ruan H (2002). A molecular approach for identification of bovine and ovine-derived materials from animalsderived feedstuffs. Chin. J. Anim. Sci. 38: 5.

Fumihito A, Miyake T, Sumi S, Takada M, et al. (1994). One subspecies of the red junglefowl (Gallus gallus gallus) suffices as the matriarchic ancestor of all domestic breeds. Proc. Na. Acad. Sci. 91: 12505-12509.

Fumihito A, Miyake T, Takada M, Shingu R, et al. (1996). Monophyletic origin and unique dispersal patterns of domestic fowls. Proc. Natl. Acad. Sci. U. S. A. 93: 6792-9795.

Gong PPC, Liu LX, Li DL, Yuan YY, et al. (2011). The investigation of genetic diversity of piao chicken based on mitochondrial DNA D-loop region sequence. J. Yunnan Agric. Univ. 26: 211-214.

He DQ, Zhu Q, Chen SY, Wang HY, et al. (2008). A homogenous nature of native Chinese duck matrilineal pool. BMC Evol. Biol. 8: 298.

Huang DP, Li DL, Yuan F, Lin C, et al. (2010). Genetic diversity of mitochondrial DNA D-loop region of Xichou blackbone chicken. J. Yunnan Agric. Univ. 25: 373-377.

Jonas E, Greger L, Ulrika G, Bertrand B, et al. (2008). Identification of the Yellow skin gene reveals a hybrid origin of the domestic chicken. PLoS Genet. 4: e1000010.

Kumar S, Tamura K and Nei M (2004). MEGA3: Integrated software for Molecular Evolutionary Genetics Analysis and sequence alignment. Brief. Bioinform. 5: 150-163.

Lai SJ, Wang L, Liu YP and Li XW. (2005). Study on mitochondrial DNA genetic polymorphism of some yak breeds in China. Coll. Anim. Sci. Tech. 32: 463-470.

Lan H, Wang W and Shi LM (1995). Genetic diversity of mitochondrial DNA sequences in swine and wild boar in Southwest. J. Genet. Genomics 1: 28-33.

Li H and Qiu YP (2003). Research status of genetic diversity of black-bone chicken. Chin. J. Anim. Sci. 39: 51-53.

Li JH and Wang JW (2007). Studies on ND4 gene polymorphism and genetic structure among domestic goose breeds. $J$. Anhui Agric. Sci. 35: 2924-2925.

Li L and Zhu Q (2006). Genetic diversity in Sichuan Black-Bone Chickens as revealed by microsatellite DNA markers. Heilongjiang Anim. Sci. Vet. Med. 2: 18-20.

Li HF, Zhu WQ, Song WT, Shu JT, et al. (2010). Origin and genetic diversity of Chinese domestic ducks. Mol. Phylogenet. Evol. 57: 634-640.

Liu YP, Wu GS, Yao YG, Miao YW, et al. (2006). Multiple maternal origins of chickens: out of the Asian jungles. Mol. Phylogenet. Evol. 38: 12-19.

Nei M (1982). Evolution of human races at the gene level. Prog. Clin. Biol. Res. 103 Pt A: 167-181.

Niu D, Fu Y, Luo J, Ruan H, et al. (2002). The origin and genetic diversity of Chinese native chicken breeds. Biochem. Genet. 40: 163-174.

Rozas J, Sánchez-DelBarrio JC, Messeguer X and Rozas R (2003). DnaSP, DNA polymorphism analyses by the coalescent and other methods. Bioinformatics 19: 2496-2497.

Sambrook J, Fritsch EF and Maniatis T (1989). Molecular Cloning: A Laboratory Manual. 2nd edn. Cold Spring Harbor Laboratory Press, New York.

Song CH, Chen HJ, Ma YH, Tang H, et al. (2007). Maternal origins of six indigenous chicken breeds in China Acta Vet. Zootechnica Sin. 38: 735-740. 
Sun GR, Zhu Q and Li L (2003). Genetic variation of nine microsatellite loci in silkies. Sichuan Anim. Vet. Sci. 30: 20.

Tang QP, Chen KW, Li HF, Zhang SJ, et al. (2005). Analysis of genetic differentiation of Chinese game chicken by using microsatellite. J. Northwest Sci-Tech Univ. Agric. For. (Nat. Sci. Ed.) 33: 19-23.

Tang QP, Chen KW, Li HF, Zhang SJ, et al. (2005). Analysis of the genetic diversity of 12 Chinese indigenous black-bone Chicken breeds using microsatellite marker. Acta Vet. Zootechnica Sin. 36: 755-760.

Wang JW, Qiu XP, Zeng FT, Shi XW, et al. (2005). Genetic differentiation of domestic goose breeds in China. Yi Chuan Xиe Bao 32: 1053-1059.

Xiang YJG, Yang L and Zhang YP (2000). Sequence divergence between Chrysolophus amherstiae and Chrysolophus pictus. Hereditas 22: 225-228.

Yang ZQ and Liu YP (2004). Analysis of partial mtDNA sequences on Tianfu Wugu and Guizhou Mountain Wugu Chicken. Sichuan Anim. Vet. Sci. 31: 23-24.

Zhang XQ, Lu XM, Yang YH, Liu JS, et al. (1998a). Population genetic variability of microsatellite polymorphisms and RAPDs in Chinese chicken breeds in Guangdong. Acta Genet. Sin. 25: 112-119.

Zhang XY (1998b). Analysis on genetic constitution of population in black-bone chicken breeds by RAPD technique. China Poultry 20: 1-2.

Zhou Y, Zhu Q and Li L (2006). Analysis on genetic diversity of silky fowl with multiplex PCR. Hubei Agr. Sci. 45: 546-548.

Zhu Q and Li L (2003). Genetic diversity in black-bone chicken lines as revealed by microsatellite DNA markers. Acta Vet. Zootechnica Sin. 34: 213-216.

Zhu WQ, Li HF, Guo J, Song WT, et al. (2009). Analysis on the genetic diversity and the origin of mtDNA in Chinese game chicken. Acta Vet. Zootechnica Sin. 40: 1560-1563.

Zhu WQ, Li HF, Song WT, Xu WJ, et al. (2010). Analysis on systematic evolution of Chinese native light-type goose breeds based on mtDNA D-loop sequences. Acta Ecologiae Animalis Domastici 29: 549-553. 